\title{
Methodological study and a recommended technique for determining the euglobulin lysis time
}

\author{
R. CHAKRABARTI, M. BIELAWIEC,1 J. F. EVANS, AND \\ G. R. FEARNLEY
}

From the Gloucestershire Royal Hospital, Gloucester

SYNOPSIS The euglobulin lysis time, which is widely used to measure blood fibrinolytic activity, $\frac{\sim}{\sigma}$ is shown to be sensitive to the temperature and $p \mathrm{H}$ at which the euglobulin fraction of plasma is 8 prepared and also to the type of anticoagulant employed and buffer in which it is suspended. $\mathrm{A}_{0}^{\infty}$ standardized method for performing the test, which controls these variables and has been found to $\frac{}{3}$ yield reproducible results, is described. Since, at present, data obtained with this test by different $\vec{Z}$ laboratories are, for the reasons given, rarely comparable, it is hoped that the method outlined may be considered suitable for general adoption.

The natural or spontaneous fibrinolytic activity of blood can be measured by the time required for lysis of clots made from dilute plasma (Biggs, MacFarlane, and Pilling, 1947; Fearnley and Tweed, 1953), dilute blood (Fearnley, Balmforth, and Fearnley, 1957), or the euglobulin fraction of plasma (various authors). The latter is believed to measure the plasminogen activator activity of the blood sample, unimpeded by the presence of plasmin inhibitors. The euglobulin fraction contains nearly all the plasminogen, all the plasminogen activator, and about $20 \%$ of the fibrinogen of the parent plasma, antiplasmin remaining in the supernatant (Kowalski, Kopec, and Niewiarowski, 1959).

The euglobulin fraction is prepared by acidification of plasma, diluted with distilled water, either by the addition of acetic acid (Milstone, 1941) or of carbon dioxide (von Kaulla and Schultz, 1958). Blix (1961) has shown that variations in the technique will markedly affect the results of the test; and it is evident from the literature that the range of euglobulin lysis time varies with different workers, that such variations reflect differences of technique, and that results obtained by different laboratories are, therefore, rarely comparable.

\section{MATERIAL AND METHODS}

SUBJECTS Healthy subjects and patients with occlusive vascular disease.

ACETIC ACID REAGENT A stock solution of $1 \%$ concen-

${ }^{1}$ Of the Medical Academy, Bialystok, Poland.

Received for publication 21 March 1968. tration made from anhydrous acetic acid in distilled $\vec{\bullet}$ water, from which appropriate dilutions were made.

\section{ANTICOAGULANTS}

1 Sodium citrate $3.8 \%$ in distilled water.

2 Potassium oxalate $4.0 \%$ in distilled water

3 Dipotassium - ethylene - diamine - tetra - acetic acid (EDTA) $2.25 \%$ in distilled water

4 Heparin 25 units $/ \mathrm{ml}$ in physiological saline (Heparin B.P., Evans Medical Ltd).

BUFFERS

1 Phosphate buffer, $p \mathrm{H} 7 \cdot 4$ (Fearnley et al, 1957)

2 Barbitone buffer $p \mathrm{H} \mathrm{7.35}$ (Nilsson and Clow, 1962)

3 Saline phosphate buffer $(p \mathrm{H} 7 \cdot 4)$ made by combiningo 1 part phosphate buffer (1) with 4 parts physiologicalosaline solution.

4 Acetate buffer, sodium acetate $0 \cdot 12 \mathrm{M}=p \mathrm{H} 7 \cdot 4$.

THROMBIN Parke Davis \& Co. in saline diluted to 5 units per $0.1 \mathrm{ml}$

$p$ H Meter Pye model 79.

Venous blood was obtained without stasis, using a no. 1 stainless steel needle and a siliconized syringe. Ito was mixed with the appropriate anticoagulant and $\mathrm{N}$ centrifuged immediately in an angle centrifuge at 3,000요 rpm for 10 minutes to obtain the plasma.

PREPARATION OF EUGLOBULIN FRACTION Two methods were used. (1) One part plasma was mixed with 19 parts dilute acetic acid. Ten minutes later the resulting pre- $\mathbb{D}$ cipitate was separated by centrifugation at $2,000 \mathrm{rpm}^{-}$for six minutes. (2) One part plasma was mixed with $10 \frac{0}{\circ}$ parts distilled water in a conical flask of $50 \mathrm{ml}$ capacity. $\frac{\mathrm{O}}{\Phi}$ Carbon dioxide was blown over the surface of the mixture for three minutes while the flask was being $\vec{Q}$ rotated. The resulting precipitate was separated by centrifugation as above. 
MEASUREMENT OF LYSIS TIME In both methods the supernatant was discarded and the inner surface of the centrifuge tube was dried with filter paper. The precipitate was then suspended in a volume of the appropriate buffer equal to twice that of the plasma from which it was obtained. Equal volumes of the suspension were transferred to two test tubes of dimensions 5 in. $\times \frac{1}{2}$ in., and clotted by the addition of 5 units of topical thrombin. The tubes were then incubated in a Perspex water bath at $37^{\circ} \mathrm{C}$, and the time required for complete dissolution of the clot, measured to the nearest quarter of an hour, was recorded as the lysis time.

ESTIMATION OF FIBRINOGEN Fibrinogen was measured gravimetrically after conversion to fibrin according to Fearnley and Chakrabarti (1966).

\section{METHODOLOGICAL INVESTIGATIONS}

EFFECT OF TEMPERATURE Fibrinolytic activity is labile in fluid blood and plasma kept at room temperature, but its loss can be minimized by keeping blood samples at low temperature (Fearnley, Revill, and Tweed, 1952). The labile activator is stabilized by adsorption to fibrin when clotting takes place (Fearnley, 1953). On the other hand, incubation of the euglobulin fraction of plasma in glass has been reported to increase its fibrinolytic activity (Iatridis and Ferguson, 1961). (Observations made by us indicate that some increase of fibrinolytic activity occurs when the euglobulin fraction is incubated even in siliconized or plastic containers.) Temperature, therefore, has opposite effects on fibrinolytic activity in plasma and its euglobulin fraction.

These effects of temperature were studied in 10 subjects. Euglobulin precipitation was by carbon dioxide.

(a) Each step of the test, viz collection of blood, centrifugation, precipitation of the euglobulin fraction, its centrifugation and suspension in buffer, and its coagulation with thrombin was done at room temperature $\left(22\right.$ to $\left.24^{\circ} \mathrm{C}\right)$.

(b) Blood was collected and centrifuged and the euglobulin fraction precipitated at room temperature. The euglobulin precipitate was separated by centrifugation at $4^{\circ} \mathrm{C}$, re-suspended in buffer and clotted with thrombin at the same temperature.

(c) After collection, blood was immediately cooled to $4^{\circ} \mathrm{C}$ and every step, until incubation of the clot, was carried out at this temperature.

Table I gives the results. It can be seen that on every occasion except one (no. 4), the lysis times obtained with technique $(c)$ were shorter than those obtained with techniques $(a)$ and $(b)$, indicating that maximal activator activity was preserved by this technique $(c)$. The results obtained with technique
TABLE I

EFFECT OF TEMPERATURE DURING PREPARATION OF EUGLOBULIN FRACTION IN 10 SUBJECTS USING THREE METHODS AND COMPLETE DISSOLUTION OF THE CLOT EXPRESSED AS LYSIS TIME IN HOURS

\begin{tabular}{llll}
$\begin{array}{l}\text { Subject } \\
\text { No. }\end{array}$ & \multicolumn{2}{l}{ Experiment } \\
\cline { 2 - 4 } & $a(h r)$ & $b(h r)$ & $c(h r)$ \\
\hline 1 & 4 & $5 \frac{1}{2}$ & $2 \frac{1}{2}$ \\
2 & $4 \frac{1}{2}$ & 3 & $1 \frac{1}{2}$ \\
3 & $1 \frac{1}{2}$ & $2 \frac{1}{2}$ & $4 \frac{1}{2}$ \\
4 & $3 \frac{1}{2}$ & $5 \frac{1}{2}$ & 3 \\
5 & $3 \frac{1}{2}$ & $5 \frac{1}{2}$ & $1 \frac{1}{2}$ \\
6 & $1 \frac{1}{2}$ & 2 & $3 \frac{1}{2}$ \\
7 & $4 \frac{1}{2}$ & $6 \frac{3}{2}$ & $1 \frac{1}{4}$ \\
8 & $2 \frac{1}{2}$ & $3 \frac{1}{2}$ & $1 \frac{1}{2}$ \\
9 & 2 & $3 \frac{1}{2}$ &
\end{tabular}

(a) suggest that loss of activator in blood and plasma at room temperature was partially compensated by increase of activator activity in the euglobulin fraction caused by contact with glass at room temperature. The results obtained with technique $(b)$ suggest that increase of activator activity in the euglobulin fraction due to contact with glass was minimized by keeping this fraction at low temperature until the clots were formed.

The results of the experiment are thus compatible with a dual effect of temperature on fibrinolytic activity in respect of plasma and its euglobulin fraction.

EFFECT OF ANTICOAGULANTS The effect of four anticoagulants was studied in each of seven subjects, using a low temperature technique throughout. To aliquots of blood from each subject, sodium citrate and sodium oxalate solutions were added in two concentrations 1:5 and 1:10 and EDTA and heparin solutions in a concentration of $1: 10$. Table II gives the mean lysis time of the seven subjects. It can be seen that the shortest lysis times were obtained with sodium citrate, and that when the concentration of this salt was $1: 5$, the mean lysis time $(2 \cdot 7$ hours $)$

TABLE II

EUGLOBULIN LYSIS TIME (MEAN OF SEVEN RESULTS) USING DIFFERENT ANTICOAGULANTS ${ }^{1}$

\begin{tabular}{lcc} 
Anticoagulant & $\begin{array}{l}\text { Ratio of } \\
\text { Anticoagulant }: \text { Blood }\end{array}$ & $\begin{array}{l}\text { Euglobul } \\
\text { Lysis Tim }\end{array}$ \\
\hline Sod. citrate & $1: 4$ & 2.7 \\
& $1: 9$ & 4.0 \\
Pot. oxalate & $1: 4$ & 4.4 \\
& $1: 9$ & 4.9 \\
EDTA & $1: 9$ & 6.3 \\
Heparin & $1: 9$ & 6.8
\end{tabular}

${ }^{1}$ Euglobulin precipitated by carbon dioxide. 
was considerably shorter than that (4.0 hours) when its concentration was $1: 10$. This confirms Buckell's (1958) observation. In contrast to sodium citrate, there was less appreciable difference of mean lysis time with the two concentrations of sodium oxalate used, EDTA and heparin gave the longest mean lysis times.

EFFECT OF $p \mathrm{H}$ A constant $p \mathrm{H}$ of 5.4 is obtained when the euglobulin fraction is precipitated with carbon dioxide, whereas the $p \mathrm{H}$ obtained with acetic acid precipitation varies somewhat. To investigate the influence of $p \mathrm{H}$ during preparation of the euglobulin fraction on lysis time, different concentrations of acetic acid were used to precipitate the euglobulin fraction from aliquots of the plasma of one subject. This was repeated in six subjects. At the same time, the amount of clottable fibrinogen precipitated was determined on each occasion. The results are shown in Table III, where it can be seen that $p \mathrm{H}$ exercises a biphasic effect on lysis time. This effect is reminiscent of that observed by Fearnley and Lackner (1955) using dilute plasma clots. Table III also shows that the amount of clottable fibrinogen precipitated rose with increasing $p \mathbf{H}$ to reach a peak and thereafter fell. These results confirm those of Blix (1961).

\section{TABLE III}

EUGLOBULIN PRECIPITATED AT DIFFERENT $p H$ BY DIFFERENT DILUTIONS OF ACETIC ACID IN SIX SUBJECTS

Acetic Acid Dilution

\begin{tabular}{llll}
\hline $1: 120 \quad 1: 240 \quad 1: 360 \quad 1: 428$
\end{tabular}

Subject 1

pH of precipitation

Fibrinogen (mg)

ELT

$4 \cdot 6$
20
14

$5 \cdot 1$
40
$2 \frac{1}{2}$

$\begin{array}{cc}5 \cdot 4 & 5 \cdot 9 \\ 100 & 140 \\ 1 \frac{1}{2} & 1\}\end{array}$

Subject 2

pH

Fibrinogen (mg)

ELT

$4 \cdot 8$
40
$3 \frac{1}{2}$

$5 \cdot 3$
120
$4 \frac{1}{2}$

$\begin{array}{cc}5 \cdot 7 & 6 \cdot 1 \\ 240 & 220 \\ 39 & 2 ?\end{array}$

Subject 3

pH

Fibrinogen (mg)

ELT

$4 \cdot 9$
122

$5 \cdot 4$
210
28

$\begin{array}{cc}5 \cdot 9 & 6 \cdot 15 \\ 240 & 200 \\ 4 \downarrow & 2 \frac{1}{2}\end{array}$

Subject 4

pH

Fibrinogen (mg)

ELT

$4 \cdot 7$
100
$1 \frac{1}{2}$

$5 \cdot 2$
210
2

$5 \cdot 7$

320

Subject 5

Fibrinogen (mg)

ELT

$4 \cdot 7$
260
$2 t$

$5 \cdot 1$
300
3

$5 \cdot 7$

380

420

Subject 6

$p \mathrm{H}$

Fibrinogen (mg)

ELT

$\begin{array}{cccc}4 \cdot 7 & 5 \cdot 25 & 5 \cdot 85 & 6 \cdot 0 \\ 40 & 150 & 240 & 160 \\ 2 & 3 \downarrow & 2 \frac{1}{2} & 2\end{array}$

EFFECT OF BUFFERS The effect of resuspending euglobulin precipitates obtained at constant $p \mathrm{H}$ b carbon dioxide in barbitone, saline phosphate, an $\Phi$ acetate buffers was studied in five subjects. Table IT shows that the lysis times were shortest when res suspended in acetate buffer, were longest in barbi tone buffer, while those in phosphate saline buffees were intermediate.

TABLE IV

EUGLOBULIN LYSIS TIME

Acetate Buffer Phosphate Saline

\begin{tabular}{|c|c|c|c|}
\hline $\begin{array}{l}\text { Subject } 1 \\
\text { Subject } 2 \\
\text { Subject } 3 \\
\text { Subject } 4 \\
\text { Subject } 5\end{array}$ & $\begin{array}{l}2 \frac{1}{2} \mathrm{hr} \\
4 \mathrm{hr} \\
1 \frac{3}{\mathrm{hr}} \\
2 \frac{3}{\mathrm{hr}} \\
2 \frac{1}{2} \mathrm{hr}\end{array}$ & $\begin{array}{l}28 \mathrm{hr} \\
6 \mathrm{hr} \\
2 \mathrm{t} \mathrm{hr} \\
3 \mathrm{t} \mathrm{hr} \\
3 \mathrm{hr}\end{array}$ & $\begin{array}{l}3 \mathrm{hr} \\
8 \mathrm{hr} \\
2 \mathrm{hr} \\
3 \mathrm{hr} \\
4 \mathrm{hr}\end{array}$ \\
\hline
\end{tabular}

\section{DISCUSSION}

The euglobulin lysis time is probably the mos specific method for measuring spontaneous fibrino $\vec{\oplus}$ lytic activity in that it has the advantage over the dilute blood clot lysis time (Fearnley et al, 1957) of estimating the level of activator activity in the absence of antiplasmin. In contrast to the dilutes blood clot lysis time, however, which a survey of the literature shows to give comparable results in the hands of different workers, euglobulin lysis times obtained by different laboratories using ostensibl $\vec{b}$ the same technique vary considerably.

We believe that the greatest source of such variation is failure to take account of the lability of plas? minogen activator in blood and plasma at roonto temperature (Fearnley et al, 1952). It was in facf recognition of this lability which led to the demon 3 stration that spontaneous fibrinolytic activity is property of the blood of unstressed people (Fearnley and Tweed, 1953), and is not dependent on violens exercise or the injection of adrenaline for its ap? pearance, as was previously thought (Biggs et at 1947). Cooling blood samples to $4^{\circ} \mathrm{C}$ will greatl reduce but not entirely prevent loss of activator ando in our experience, preparation of the euglobulin fraction should be started within a few minutes of obtaining blood, using a low temperature technique throughout, if accurate and reproducible results are to be obtained. The experiments presented were designed to show the importance of temperatur\& control, not only in the preservation of fibrinolytic activity in blood and plasma, but also in the preven tion of the increase of activator activity caused b. glass contact.

Carbon dioxide precipitation, according to th尺 method of von Kaulla and Schultz (1958), has the 
great advantage of ensuring a uniform $p \mathrm{H}$ of $5 \cdot 4$, whereas acetic acid precipitation results in a variable $p H$. Our data confirm Blix's (1961) findings that the lysis time of the euglobulin fraction is affected by the $p \mathrm{H}$ at which it is prepared and that this may be partly or solely a function of the amount of fibrinogen precipitated from plasma. For this reason we believe that carbon dioxide is preferable to acetic acid as a precipitant.

Our data also indicate that the euglobulin lysis time is affected by the anticoagulant used and, in the case of citrate, by its concentration, as was first reported by Buckell (1958). Buffers used also markedly affect the lysis time, as our data show.

A survey of the literature shows that euglobulin lysis times of healthy people reported by different laboratories range from one to 10 hours, or even longer. Using von Kaulla's method and rigid low temperature technique, our experience over the last five years is that the lysis times of healthy adults lie between three-quarters of an hour and three hours. Furthermore, such times are closely reproducible for the individual. The main object of this communication is to put forward a plea that the euglobulin lysis time be standardized. Our experience indicates that comparable results will be obtained with this test if von Kaulla's method is carried out as follows:

\section{RECOMMENDED TECHNIQUE}

Blood is collected without venous stasis by clean venepuncture and immediately mixed with $3.8 \%$ sodium citrate solution in a test tube standing in ice and water, the proportion of blood to anticoagulant being $4: 1$. The plasma is separated in a refrigerated centrifuge at
$3,000 \mathrm{rpm}$ for 10 minutes. One ml plasma is added to $10.0 \mathrm{ml}$ distilled water in a conical flask of $50 \mathrm{ml}$ capacity standing in a beaker of ice and water. Carbon dioxide is blown over the surface of the mixture for three minutes by means of a Pasteur pipette connected by rubber tubing and valve to the delivery cylinder. During this procedure, the flask is gently rotated to ensure full exposure of the mixture to the gas.

The mixture is then transferred to a centrifuge, to be centrifuged at $4^{\circ} \mathrm{C}$ at $2,000 \mathrm{rpm}$ for six minutes. The supernatant is discarded and the inner surface of the tube dried with no. 1 Whatman filter paper. The euglobulin precipitate is resuspended in $2.0 \mathrm{ml}$ of saline phosphate buffer. The suspension is equally divided into two test tubes 5 in. $\times \frac{1}{2}$ in. and is clotted with 5 units $(0.1 \mathrm{ml})$ thrombin in saline. The whole procedure is done in an ice water bath. After five minutes the tubes are transferred to a water bath at $37^{\circ} \mathrm{C}$ and the time taken by the clot to dissolve completely is read as the lysis time.

This study was supported by grants from the Nuffield Foundation, Bayer Products Ltd, and Organon Laboratories Ltd.

\section{REFERENCES}

Biggs, R., Macfarlane, R. G., and Pilling, J. (1947). Lancet, 1, 402.

Blix, S. (1961). Scand. J. clin. Lab. Invest., 13, suppl. 58.

Buckell, M. (1958), J. clin. Path., 11, 403.

Fearnley, G. R., Revill, R., and Tweed, J. M. (1952). Clin. Sci., 11, 309.

- , and Tweed, J. M. (1953). Ibid., 12, 81.

(1953). Nature (Lond.), 172, 544.

and Lackner, R. (1955). Brit. J. Haemat., 1, 189

—, Balmforth, G., and Fearnley, E. (1957). Clin. Sci., 16, 645. and Chakrabarti, R. (1966). Lancet, 2, 757.

Iatridis, S. G., and Ferguson, J. H. (1961). Thrombos. Diathes. haemorrh. (Stuttg.), 6, 411.

Kowalski, E., Kopec, M., and Niewiarowski, S. (1959). J. clin. Path., 12, 215.

Milstone, H. (1941). J. Immiunol., 42, 109.

Nilsson, I. M., and Clow, B. (1962). Acta chir. Scand., 123, 247.

von Kaulla, K. N., and Schultz, R. L. (1958). Amer. J. clin. Path., 29, 104. 